\title{
Correlation between the Characteristic Flavour of Youtiao and Trans Fatty Acids Assessed via Gas Chromatography Mass Spectrometry and Partial Least Squares Regression Analyses
}

\author{
Xuelian Yang, ${ }^{1,2,3}$ Xiangyu Zhang, ${ }^{3}$ Jianchun Xie $\mathbb{C D}^{1,2,3}$ Dongdong Yang, \\ and Chengtao Wang ${ }^{1,2,3}$ \\ ${ }^{1}$ Beijing Advanced Innovation Center for Food Nutrition and Human Health, Beijing Technology \& Business University (BTBU), \\ Beijing 100048, China \\ ${ }^{2}$ Beijing Engineering and Technology Research Center of Food Additives, Beijing Technology \& Business University (BTBU), \\ Beijing 100048, China \\ ${ }^{3}$ Beijing Technology \& Business University (BTBU), Beijing 100048, China
}

Correspondence should be addressed to Jianchun Xie; xjchunbtbu@163.com

Received 22 May 2020; Revised 14 September 2020; Accepted 17 September 2020; Published 29 September 2020

Academic Editor: Jesús Lozano

Copyright $\odot 2020$ Xuelian Yang et al. This is an open access article distributed under the Creative Commons Attribution License, which permits unrestricted use, distribution, and reproduction in any medium, provided the original work is properly cited.

\begin{abstract}
This study aimed to analyse trans fatty acid (TFA) levels and key volatile flavour substances in fried youtiao prepared using five common edible oils and the relationship between TFAs and key volatile flavour substances via partial least squares regression (PLSR) analysis. Total TFA levels were the highest on using rapeseed oil during frying (approximately $1.061 \mathrm{mg} / \mathrm{g}$ ), probably owing to the high content of unsaturated fatty acids in rapeseed oil and their instability. In total, 22 key flavour substances were detected. Although the flavours differed with different oils, flavour compounds including 3-(methyl sulphide) propionic aldehyde, (E,E)2,4-sebacedienal, nonaldehyde, and 3-hydroxy-2-butanone contributed to overall flavour. PLSR analysis revealed that C18:2, 9t12t is produced with (E)-2-hexenaldehyde and nonaldehyde. (E,E)-2,4-sebacedienal levels were positively correlated with those of C18:2, 9c12t and C18:2, 9t12c. Most aliphatic aldehydes and pyrazines yield C18:3, 9t12t15c TFAs. These results indicate the characteristic flavour profile of youtiao and promote the preparation of healthy fried food.
\end{abstract}

\section{Introduction}

Youtiao, a Chinese traditional fried breakfast item with a long history, has a characteristic crispy covering, a soft and flavourful interior, a golden colour, and an appealing aroma. It is popular among different groups and is a prominent aspect of the Chinese food culture $[1,2]$. Nowadays, youtiao is extensively served as a breakfast item in fast food restaurants. Youtiao is popular not only in China but also in Indonesia, Laos, Myanmar, and other Southeast Asian countries $[3,4]$.

Various types of trans fatty acids (TFAs) derived from fried food have received increasing attention among consumers, especially regarding their potential health hazards in humans and their potential to increase the risk of coronary heart disease, breast cancer, and type II diabetes. According to the guidelines of the $\mathrm{WHO}$ and numerous institutions including the FDA, trans isomers of linoleic acid and their association with the risk of the aforementioned diseases are apparent, and TFAs are speculated to be formed primarily owing to isomerization of unsaturated fatty acids during high-temperature frying $[5,6,7,8]$. Simultaneously, flavour is a major deciding factor of food preferences among consumers. Studies on food flavour have made significant advancements thus far. For example, Lotfy, Fadel, ElGhorab, and Shaheen et al. $[9,10,11,12]$ assessed the oil used for frying and the changes in its composition and characteristics, reporting that oil fried at 170,180 , and $190^{\circ} \mathrm{C}$ for $70 \mathrm{~h}$ had increased levels of TFAs, potentially undergoing isomerization. It has been reported that the primary volatile 
flavour substances in bread bran were heptaldehyde, 3methyl-1-butanol, 2-methyl-pyrazine, 2,5-dimethylpyrazine, 2,3-dimethylpyrazine, 2-acetylpyrazine, and 4-hydroxyl-2,5-dimethyl-3-hydrofuranone [6, 13, 14, 15, 16, 17]. The flavouring substances in steamed bread have been analysed, and it has been reported that the primary flavouring substances are ethanol, phenylethanol, and 3methyl butanol [7, 18-21]. Headspace solid phase microextraction (HS-SPME) along with gas chromatography mass spectrometry (GC-MS) analysis was carried out, and it was reported that the primary characteristic flavour compounds of youtiao are (E,E)-2,4-sebacedienal, 3-methyl butyraldehyde, 1-octene-3-alcohol, furfural, and furfuryl alcohol $[10,22,23]$. However, few studies have reported methods of retaining the relevant flavours while simultaneously decreasing the TFA content, thus promoting the preparation of healthy and flavourful fried food, which has long been desirable among consumers. This study investigated the association between the characteristic flavour substances in youtiao and TFAs.

Formation of flavour substances is significantly associated with cracking of oil. This study first analysed the TFA content using different cooking oils to fry youtiao and identified the flavour substances in youtiao via GC-MS/GColfactometry analysis; thereafter, PLSR analysis was performed to explore the correlation between the key volatile compounds and TFA levels. The composition of characteristic flavour substances in youtiao and the flavourimparting mechanism can be considered a reference for retaining key flavour substances and further controlling the formation of TFAs, thus promoting the preparation of healthy fried food items.

\section{Materials and Methods}

2.1. Materials. Flour was obtained from Henan Xuejian Industrial Co., LTD.; sunflower oil and soybean oil, COFCO Fulinmen Food Marketing Co., LTD; rapeseed oil, China Grain Storage Zhenjiang Grain and Oil Co. LTD.; palm oil, Tianjin Julong Grain and Oil Co. LTD.; peanut oil, Shandong Luhua Group Co. LTD.; leavening agent, Hubei Yichang Anqi Yeast Co. LTD. and Beijing Salt Co., LTD; white granulated sugar, China Sugar and Alcohol Group Corporation; 1,2-O-dichlorobenzene (99\%) and C5-C25 n-alkanes (chromatographically pure), Beijing Chemical Reagent Co., LTD.; mixed standard and flavour compound of methyl oleate and TFA methyl ester, Sigma-Aldrich, Darmstadt, Germany; and isooctane, dichloromethane, and anhydrous sodium sulphate (chromatographically pure) were purchased from Sinopac Chemical Reagent Co., LTD.

2.2. Sample Preparation. Three hundred grams of flour, $12 \mathrm{~g}$ leavening agent, $4.5 \mathrm{~g}$ sugar, $3 \mathrm{~g}$ salt, and $210 \mathrm{~g}$ deionized water, mixed into the dough, sealed in plastic wrap, and the dough was maintained in a $28^{\circ} \mathrm{C}$ water bath for $30 \mathrm{~min}$. The dough was then uncovered, spread with oil, and 3-10 cmthick pieces were cut out. Two pieces were fold together, $500 \mathrm{~mL}$ of fresh vegetable oil was added in a pan preheated to $210^{\circ} \mathrm{C}$, and the dough was fried, thus yielding youtiao, and the entire process of frying was carried out at $190 \pm 10^{\circ} \mathrm{C}$, after which the fried youtiao was allowed to drain from both sides (approximately 2 3 $\mathrm{min}$ ). Thus, 13-14 pieces of youtiao were prepared in approximately $45 \mathrm{~min}$. The aforementioned steps were repeated with different types of oils and fried in 3 parallel sets.

2.3. Analysis of TFAs. Accurately, in accordance with the sample, $60.0 \mathrm{mg}$ can be accommodated in the test tube. Four millilitres of isooctane (methyl heptadecanoate, $2.5 \mathrm{~g} / \mathrm{L}$ ) was added to dissolve the sample, and $200 \mu \mathrm{L}$ potassium hydroxide in methanol was added, after which the tube was plugged with a glass stopper, oscillate on the shaker for 2 min, and allow the mixture to clarify. Thereafter, approximately $1 \mathrm{~g}$ sodium bisulphate was added, the tube was agitated for $1 \mathrm{~min}$, neutral potassium hydroxide was added, and the mixture was allowed to stand for clarification, after filtering the mixture through a $0.22 \mu \mathrm{m}$ membrane filter in sample bottles for GC analysis. Three samples were prepared in parallel [24].

Using the Gas Chromatography Agilent 7890B system and CP Sil-88 columns $(100 \mathrm{~m} \times 0.2 \mathrm{~mm} \times 0.25 \mu \mathrm{m})$, the initial oven temperature was maintained at $45^{\circ} \mathrm{C}$ for $4 \mathrm{~min}$, $13^{\circ} \mathrm{C} / \mathrm{min}$ to $175^{\circ} \mathrm{C}$ for $27 \mathrm{~min}$, and $4^{\circ} \mathrm{C} / \mathrm{min}$ to $215^{\circ} \mathrm{C}$ for $29 \mathrm{~min}$, followed by $220^{\circ} \mathrm{C}$ after $2 \mathrm{~min}$. An FID detector (detector temperature, $300^{\circ} \mathrm{C}$ ) was used, and the carrier gas was highly pure $\mathrm{N}_{2}$ with a flow rate of $1.0 \mathrm{~mL} / \mathrm{min}$. The sample injection port was set to $250^{\circ} \mathrm{C}$, with a split ratio of $50: 1$ and sample volume, $1 \mu \mathrm{L}$.

2.4. Extraction of Volatile Flavour Compounds. Fried youtiao was cut into $0.5 \times 0.5 \mathrm{~cm}^{2}$ pieces and mixed well, and $150 \mathrm{~g}$ of pieces was placed in a $1 \mathrm{~L}$ beaker. Thereafter, dichloromethane (heavy steam) was used for continuous extraction thrice at boiling point temperature $(3 \times 450 \mathrm{~mL})$. During extraction, the mixture was continuously stirred. The extraction liquid $(2 \times 150 \mathrm{~g})$ of youtiao was combined. To set the extract for SAFE device processing, a water tank was used at a constant temperature and distillation head with water at $25^{\circ} \mathrm{C}$, liquid was distilled using liquid nitrogen for cooling, and the entire distillation process was carried out to maintain the pressure at $10^{-4} \sim 10^{-5} \mathrm{~Pa}$. The distilled liquid was dried with anhydrous $\mathrm{Na}_{2} \mathrm{SO}_{4}$. After drying, the Vigreux column was used to initially concentrate the mixture to $5 \mathrm{~mL}$, and the nitrogen blowing apparatus was used to concentrate the mixture to $0.5 \mathrm{~mL}$ for GC-MS analysis [5]. The experiment was performed in triplicate.

2.5. GC-O Analysis. The DB-5MS capillary column $(30 \mathrm{~m} \times 0.25 \mathrm{~mm} \times 0.25 \mu \mathrm{m})$ was used for GC. At an initial column temperature of $40^{\circ} \mathrm{C}$, at a rate of approximately $5^{\circ} \mathrm{C} /$ min to $180^{\circ} \mathrm{C}$, with $10^{\circ} \mathrm{C} / \mathrm{min}$ to $280^{\circ} \mathrm{C}$, the temperature was maintained after running the samples for $2 \mathrm{~min}$. The carrier gas was $\mathrm{N}_{2}$ (purity, 99.999\%); velocity, $1 \mathrm{~mL} / \mathrm{min}$; temperature of the injection port, $250^{\circ} \mathrm{C}$; and splitless sample volume, $1 \mu \mathrm{L}$. 
The humidified clean air mixes with the postcolumn effluent and enters the nose. Dichloromethane was used to dilute the sample step by step in accordance with the $2^{\text {n }}$ method, and GC-O analysis was performed. DATU 2000 high-resolution olfactometer system (DATU Inc.) was used. Four trained graduated students (two men and two women, aged 23-25) performed GC-O analyses. Each panelist sniffed for twenty minutes. When the panel did not perceive any odour, the sample was no longer diluted. The highest dilution factor is the dilution factor (FD value) of the odorant [25].

\subsection{Quantitative Analysis of Key Volatile Flavour Compounds.} Twenty-two compounds with high FD values $\left(\log _{2} \mathrm{FD} \geq 4\right)$ were quantified among the aromatic substances identified via GC-MS/GC-O/aroma extraction dilution analysis in youtiao samples. The solution and samples of each mixed standard sample were analysed, using a DB-5MS chromatographic column $(30 \mathrm{~m} \times 0.25 \mathrm{~mm} \times 0.25 \mu \mathrm{m})$. The sample volume was $1 \mathrm{~L}$, and the internal standard was $1,2-\mathrm{O}$ dichlorobenzene in GC-MS in the selected ion monitoring (SIM) mode. The concentration of 1,2-O-dichlorobenzene is $1 \mathrm{mg} / \mathrm{L}$.

2.7. Statistical Analysis. Statistical analysis was performed using Unscrambler version X 10.4 (CAMO ASA, Oslo, Norway). Analysis of the key volatile flavour substances and TFA content of 5 types of edible oils used to fry youtiao was performed, with the TFAs denoted on the $X$-axis and the content of 22 different volatile flavour substances denoted on the $Y$-axis. Using standardized variables, partial least squares regression (PLSR) analysis was performed to investigate the association between key flavour substances and TFA content.

\section{Results and Discussion}

3.1. Analysis of TFAs in Fried Youtiao Prepared Using Different Edible Oils. GC/MS was performed to quantify TFAs in fried youtiao prepared using five types of vegetable oils (Table 1).

Total levels of unsaturated fatty acids in fresh vegetable oil were in the following order: sunflower $>$ rapeseed oil$>$ soybean oil $>$ peanut oil $>$ palm oil. Regarding the levels of TFAs in fried youtiao prepared using five types of vegetable oils, rapeseed oil yielded approximately $1.061 \mathrm{mg} / \mathrm{g}$ (being the maximum), since unsaturated fatty acids in rapeseed oil are unstable and are easily obtained during high-temperature heating. Peanut oil yielded the lowest TFA levels, approximately $0.100 \mathrm{mg} / \mathrm{g}$. Although peanut oil contains more unsaturated fatty acids, it does not yield TFAs, indicating that the unsaturated double bond of fatty acids in peanut oil is more stable and less prone to isomerization during frying of youtiao. Previous studies [26] reported that although fats and oils with high unsaturated fatty acid content and food items having higher nutritional value are used, during heating, especially during frying, the unsaturated double bond is extremely unstable, thus being more likely to generate TFAs. Hence, from the viewpoint of TFA generation, peanut oil yielded the least TFAs when frying youtiao.

\subsection{Analysis of the Composition of Flavour Compounds in} Different Youtiao Samples. GC-MS/GC-O/AEDA analysis was performed to quantify the key volatile flavour substances in youtiao prepared using five different edible oils (Table 2).

For 22 types of key volatile flavour substances identified via GC-MS/GC-O/AEDA analysis, we used internal standards to accurately quantify 22 types of key volatile flavour compounds in deep-fried youtiao prepared using 5 types of vegetable oils.

Sunflower oil yielded the following 6 flavour compounds at high levels: 3-methyl butyl aldehyde (642.47 ng/g), (E)-2hexene aldehyde $(346.69 \mathrm{ng} / \mathrm{g})$, nonyl aldehyde $(182.40 \mathrm{ng} /$ g), (E,E)-2,4-sebacic olefin aldehyde (245.10 ng/g), 3-hydroxy-2-butanone $(233.16 \mathrm{ng} / \mathrm{g})$, and furfuryl alcohol $(555.63 \mathrm{ng} / \mathrm{g})$; fourteen volatile flavour compounds in sunflower oil-fried youtiao had a high FD value $\left(\log _{2} \mathrm{FD} \geq 8\right)$, primarily including furfuryl alcohol, furfuryl mercaptan, and 1-octene-3-ol. Among them, the key volatile flavour compounds, such as 3-methyl butyraldehyde, nonaldehyde, (E,E)-2,4-sebacedienal, 3-hydroxy-2-butanone, and furfuryl alcohol, had high concentrations $(100 \mathrm{ng} / \mathrm{g})$ and a high dilution factor $\left(\log _{2} \mathrm{FD} \geq 8\right)$, thus contributing significantly to the overall aroma of youtiao fried in sunflower oil. These flavour compounds primarily have the unique caramel sweet, green, grease, and roasted characteristics aroma of youtiao, which appeal to consumers.

Soybean oil yielded the following 6 flavour compounds at high levels: 3-methyl butyl aldehyde (639.76 ng/g), (E)-2hexene aldehyde $(205.89 \mathrm{ng} / \mathrm{g})$, nonyl aldehyde $(188.10 \mathrm{ng} /$ g), (E,E)-2,4-sebacic olefin aldehyde (227.84 ng/g), 3-hydroxy-2-butanone $(253.42 \mathrm{ng} / \mathrm{g})$, and furfuryl alcohol $(592.03 \mathrm{ng} / \mathrm{g})$; fourteen key volatile flavour compounds had a high $\mathrm{FD}$ value $\left(\log _{2} \mathrm{FD} \geq 8\right)$, primarily including furfuryl alcohol, 3-(methylsulphyl) propionic aldehyde, and 3-hydroxy-2-butanone. Among these, the key volatile flavour compounds, such as 3-methyl butyraldehyde, nonaldehyde, (E,E)-2,4-sebacedienal, 3-hydroxy-2-butanone, and furfuryl alcohol, had both a higher concentration $(100 \mathrm{ng} / \mathrm{g})$ and a higher dilution factor $\left(\log _{2} \mathrm{FD} \geq 8\right)$, thus contributing significantly to the overall aroma of youtiao fried in soybean oil. These flavour compounds primarily have the malt, caramel, green, grease, and roasted aroma characteristic of youtiao, which appeal to consumers.

Rapeseed oil yielded the following 6 flavour compounds at high levels: 3-methyl butyl aldehyde $(634.66 \mathrm{ng} / \mathrm{g})$, (E)-2-hexene aldehyde $(213.23 \mathrm{ng} / \mathrm{g})$, nonyl aldehyde $(106.42 \mathrm{ng} / \mathrm{g}),(\mathrm{E})-2$-decyl olefin aldehyde $(122.03 \mathrm{ng} / \mathrm{g}), 3$ hydroxy-2-butanone $(246.68 \mathrm{ng} / \mathrm{g})$, and furfuryl alcohol $(347.61 \mathrm{ng} / \mathrm{g})$; eleven of these key volatile flavour compounds had a high FD value $\left(\log _{2} \mathrm{FD} \geq 8\right)$, primarily including 3(methyl sulphide) propionic aldehyde, furfuryl mercaptan, and heptaldehyde. Among these, the key volatile flavour compounds, including 3-methyl-butyral, 3-hydroxy-2butanone, and furfuryl alcohol, had both a higher concentration $(100 \mathrm{ng} / \mathrm{g})$ and a higher dilution factor 
TABLe 1: Types and levels of trans fatty acids in youtiao prepared using five types of vegetable oils (mg/g).

\begin{tabular}{lccccrc}
\hline Vegetable oil & C18:1,9t & C18:2, 9t12t & C18:2, 9c12t & C18:2, 9t12c & C18:3, 9t,12t,15c & Total \\
\hline Sunflower oil & $0.022 \pm 0.002$ & $0.008 \pm 0.001$ & $0.380 \pm 0.006$ & $0.320 \pm 0.006$ & $0.006 \pm 0.000$ & 0.736 \\
Soybean oil & $0.009 \pm 0.001$ & $0.010 \pm 0.000$ & $0.210 \pm 0.003$ & $0.170 \pm 0.003$ & $0.410 \pm 0.002$ & 0.809 \\
Rapeseed oil & $0.061 \pm 0.005$ & $0.000 \pm 0.000$ & $0.150 \pm 0.003$ & $0.120 \pm 0.003$ & $0.730 \pm 0.003$ & 1.061 \\
Palm oil & $0.098 \pm 0.004$ & $0.005 \pm 0.000$ & $0.210 \pm 0.005$ & $0.190 \pm 0.002$ & $0.090 \pm 0.009$ & 0.593 \\
Peanut oil & $0.032 \pm 0.002$ & $0.020 \pm 0.003$ & $0.030 \pm 0.004$ & $0.008 \pm 0.001$ & $0.010 \pm 0.000$ & 0.100 \\
\hline
\end{tabular}

$\overline{\mathrm{X}} \pm \mathrm{SD}$, mean \pm standard deviation, $n=3$.

$\left(\log _{2} \mathrm{FD} \geq 8\right)$, thus contributing significantly to the overall aroma of youtiao fried in rapeseed oil. These flavour compounds primarily have the oil flavour, green flavour, caramel sweet flavour, and roasted flavour characteristic of youtiao, which appeal to consumers.

Palm oil yielded the following 6 flavour compounds at high levels: 3-methyl butyl aldehyde (747.39 ng/g), (E)-2hexene aldehyde $(201.74 \mathrm{ng} / \mathrm{g}),(\mathrm{E})$-2-nonene aldehyde (112.61 ng/g), (E)-2-decyl olefin aldehyde (166.53 ng/g), 3hydroxy-2-butanone $(296.36 \mathrm{ng} / \mathrm{g})$, and furfuryl alcohol $(644.72 \mathrm{ng} / \mathrm{g})$; fourteen of these key volatile flavour compounds had a high FD value $\left(\log _{2} \mathrm{FD} \geq 8\right)$, primarily including furfuryl alcohol, (E)-2-nonenal, and 3-hydroxy-2butanone. Among these, the key volatile flavour compounds, such as 3-methyl butyraldehyde, (E)-2-nonenal, 3-hydroxy2-butanone, and furfuryl alcohol, had both a higher concentration $(100 \mathrm{ng} / \mathrm{g})$ and a higher dilution factor $\left(\log _{2} \mathrm{FD} \geq 8\right)$, thus contributing significantly to the overall aroma of youtiao fried in palm oil. These flavour compounds primarily have the oil flavour, caramel sweet flavour, and roasted flavour characteristic of youtiao, which appeal to consumers.

Peanut oil yielded the following 7 flavour compounds at high levels: 3-methyl butyl aldehyde $(492.84 \mathrm{ng} / \mathrm{g}),(\mathrm{E})-2-$ hexene aldehyde $(270.48 \mathrm{ng} / \mathrm{g})$, nonyl aldehyde $(159.79 \mathrm{ng} /$ g), (E)-2-decyl olefin aldehyde (188.15 ng/g), (E,E)-2,4sebacic olefin aldehyde (194.41 ng/g), 3-hydroxy-2-butanone $(201.80 \mathrm{ng} / \mathrm{g})$, and furfuryl alcohol $(531.00 \mathrm{ng} / \mathrm{g})$; ten of these key volatile flavour compounds had a high FD value $\left(\log _{2} \mathrm{FD} \geq 8\right)$, primarily including furfuryl alcohol, furfuryl mercaptan, and 3-(methylene sulphide) propionic aldehyde. Among these, (E,E)-2,4-sebacedienal, 3-hydroxy-2-butanone, furfuryl alcohol, and other key volatile flavour compounds had both a higher concentration $(100 \mathrm{ng} / \mathrm{g})$ and a higher dilution factor $\left(\log _{2} \mathrm{FD} \geq 8\right)$, thus contributing significantly to the overall aroma of youtiao fried in peanut oil. These flavour compounds primarily have the fried, malt, caramel sweet, roasted, grease, and green flavours characteristic of youtiao, which appeal to consumers.

Overall, using different vegetable oils to prepare youtiao, sunflower oil-fried and soybean oil-fried youtiao contained common key flavour compounds including 3-methyl butyl aldehyde, (E)-2-hexene aldehyde, nonyl aldehyde, (E,E)-2,4sebacic olefin aldehyde, 3-hydroxy-2-butanone, and furfuryl alcohol, resulting from oil cracking during high-temperature frying, showing that the primary difference in flavour between the two types of fried youtiao is not significant. However, from the viewpoint of the FD value, youtiao fried in sunflower oil contains furfuryl thiol with a high dilution factor, while youtiao fried in soybean oil did not. Soybean oil-fried youtiao contains 3-(methyl sulphide) propanal with a high dilution factor, while youtiao fried in sunflower fried did not; hence, soybean oil-fried youtiao has a relatively stringer boiled potato-like aroma than sunflower oil-fried youtiao. Similarly, comparing rapeseed oil-fried with palm oil-fried youtiao, besides the 3-methylbutyraldehyde, (E)-2hexenal, (E)-2-decenal, 3-hydroxy-2-butanone, and furfuryl alcohol levels being higher, rapeseed oil-fried youtiao contained higher levels of nonaldehyde, while palm oil-fried youtiao contained more (E)-2-nonenal. Considering the concentration of both compounds and their FD value, the concentration of furfuryl alcohol in palm oil-fried youtiao was higher than that in rapeseed oil, and the dilution factor was greater, thus imparting a stronger caramel sweetness to the youtiao. Peanut oil-fried youtiao had the highest levels of flavour compounds than those fried in the other oils and a rich aroma, and peanut oil itself may have a certain association with the aroma.

3.3. Correlation Analysis of Volatile Components and TFA Levels in Youtiao. To determine the correlation between TFA levels and the key flavour compounds in fried youtiao, PLSR analysis was performed considering 5 types of vegetable oil-fried youtiao containing 22 types of key volatile flavour compounds and 5 types of TFAs (Figure 1), and two large oval shapes of, respectively, 50\% and 100\% accounted for the variance.

The results shown in Figure 1 indicate that levels of C18: 2, 9t12t are significantly positively correlated with those of (E)-2-hexenal and nonaldehyde, indicating that the generation of these two flavour substances with a milky flavour and sweet flavour almost inevitably leads to the generation of C18:2, 9t12t. Analysis of the mechanism underlying the generation of the two flavour compounds has been reported previously [27]. The formation of (E)-2-hexenal and nonaldehyde is derived from the thermal oxidation degradation of unsaturated fatty acids, especially linoleic acid. Therefore, it can be speculated that the key milky flavour and sweet flavour of youtiao are accompanied by the inevitable generation of C18:2, 9t12t in the oil cracking process. The FD value of (E)-2-hexenal is $2^{8}$, which plays a very important role in the formation of the flavour profile of youtiao and is an essential component of the characteristic flavour substance of youtiao. This type of flavour substance is mostly produced by polyunsaturated fatty acids via thermal oxidation or degradation and amino acid decomposition. GCMS analysis (Table 2) revealed that (E)-2-hexenal and nonyl 


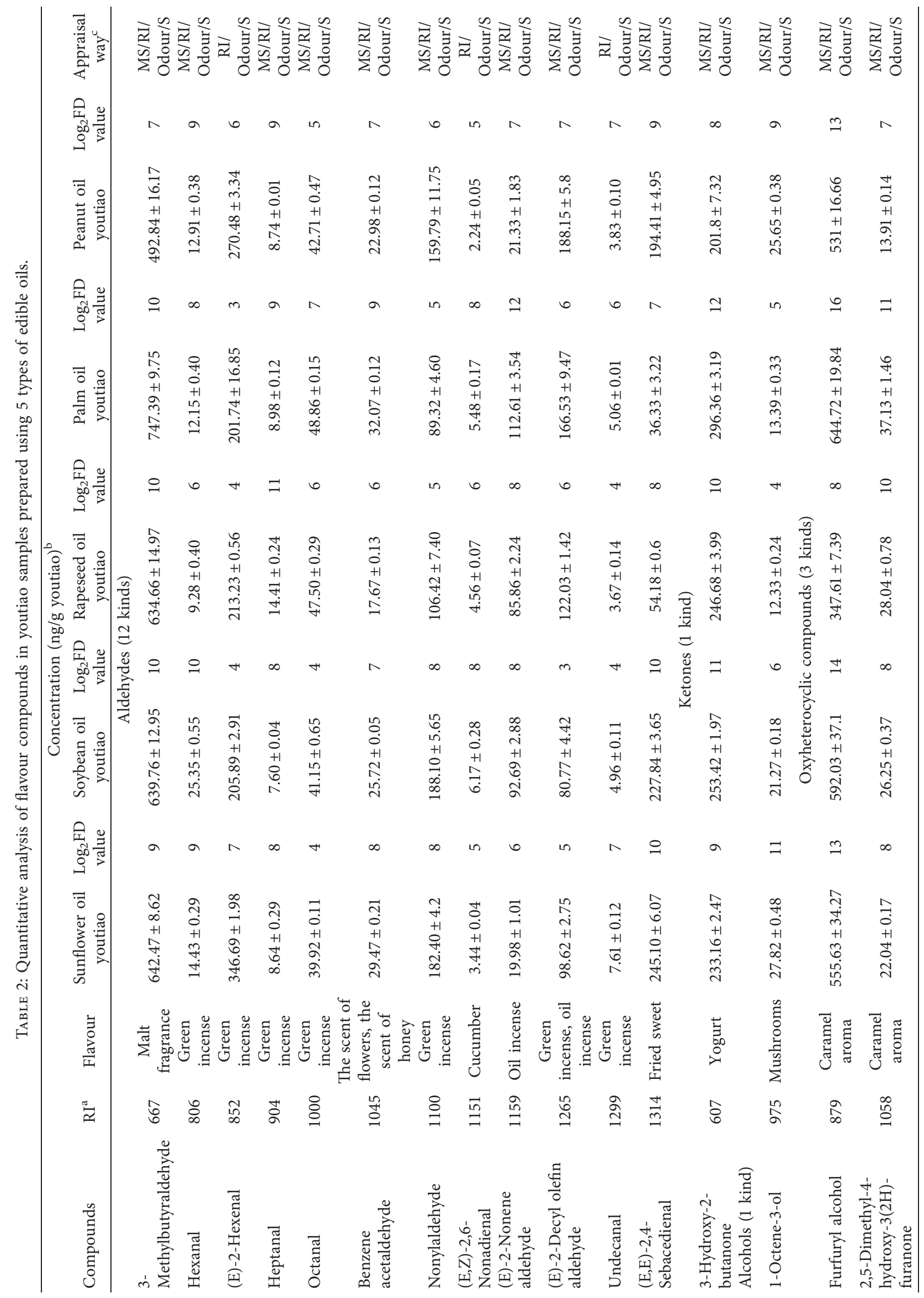




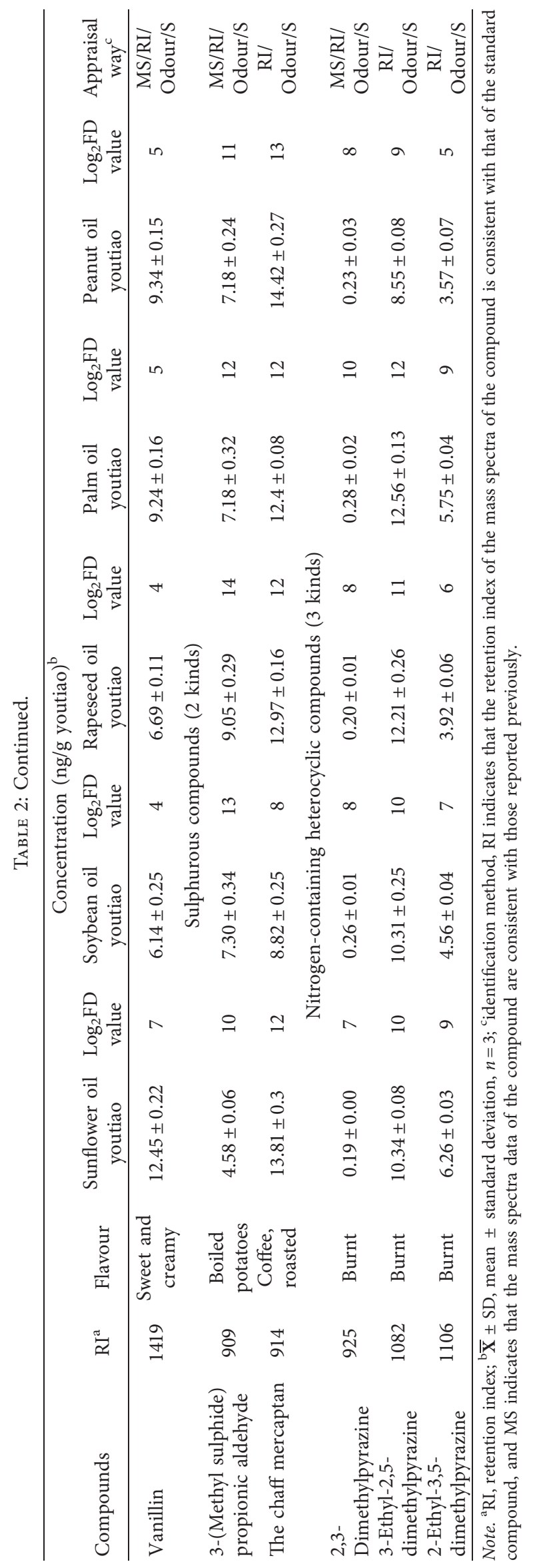




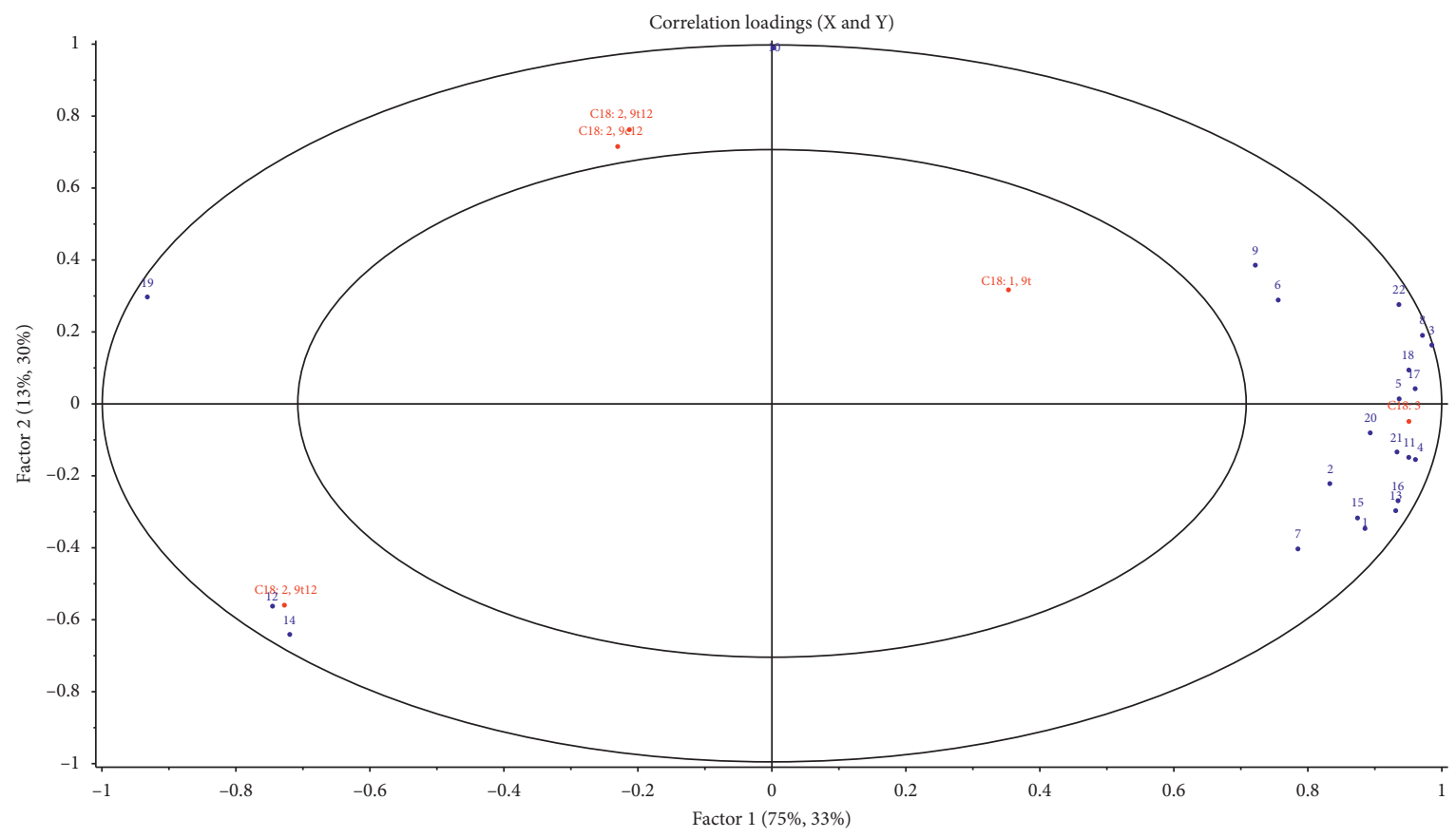

Figure 1: Analysis of the correlation between the content of trans fatty acids and the key volatile flavour substances in youtiao fried in 5 different vegetable oils. Note: the flavour substances represented by the figures are (1) hexanal; (2) heptyl aldehyde; (3) caprylic aldehyde; (4) furfuryl mercaptan; (5) 3-methylbutyraldehyde; (6) trans-2-nonenal; (7) phenylacetaldehyde; (8) trans,cis-2,6-nonadienal; (9) trans-2decenal; (10) trans, trans-2,4-sebacedienal; (11) 1-octene-3-alcohol; (12) trans-2-hexenal; (13) 3-hydroxy-2-butanone; (14) vanillin; (15) 2,3dimethylpyrazine; (16) 2-ethyl-3,5-dimethyl-pyrazine; (17) furfuryl alcohol; (18) 2,5-dimethyl-4-hydroxy-3 (2H)-furanone; (19) 3-ethyl-2,5dimethylpyrazine; (20) 3-(methyl sulphide) propionic aldehyde; (21) undecanoic aldehyde.

aldehyde generation were increased, approaching levels greater than $100 \mathrm{ng} / \mathrm{g}$, and that of oils with different C18:2, $9 t 12 \mathrm{t}$ content are generally lower, approximately $0.01 \mathrm{mg} / \mathrm{g}$, thus yielding a caramel flavour substance that does not promote C18:2, 9t12t production; if processing technology may be improved by increasing the levels of flavour substances, the accumulation of C18:2, 9t12t may be decreased.

As shown in Figure 1, (E,E)-2,4-sebacedienal and other fried flavour compounds were also formed during frying, concurrent with the preferences of consumers. Accordingly, the mechanism underlying the formation of these flavour compounds is known [2], (E,E)-2,4-sebacedienal is a hydroperoxide cracking product of 13-linoleic acid, and its levels are positively correlated with $\mathrm{C} 18: 2,9 \mathrm{c} 12 \mathrm{t}$ and $\mathrm{C} 18: 2$, 9t12c. (E,E)-2,4-Sebacic olefin aldehyde has high FD values, being not less than $2^{7}$ in all types of vegetable oils, and high levels influence the characteristic flavour profile of fried youtiao.

As shown in Figure 1, trans C18:3 levels were significantly positively correlated with fatty aldehydes such as 3methylbutyraldehyde, (E)-2-hexenal, and nonaldehyde. With 2,3-dimethyl pyrazine, 2-ethyl-3,5-dimethyl-pyrazine, furfuryl alcohol, ethyl-3-2,5-dimethyl pyrazine, 3-(methyl sulfonium) propionaldehyde, 11-carbon aldehydes, and chaff mercaptan are related, since these aldehydes are associated with the sweet malt fragrance, fresh scent, oil, and the roasted aroma, resulting from trans $\mathrm{C} 18: 3$, and 3methylbutyraldehyde and 2-ethyl-3, 5-dimethyl-pyrazine are closely related to trans $\mathrm{C} 18: 3$, based on the mechanism underlying their formation [28]. Most of the aforementioned compounds are hydroperoxide pyrolysis products of linolenic acid, among which 3-methylbutyl aldehyde and furfuryl alcohol have the highest FD value and concentration. They are essential components of the characteristic flavour of youtiao, primarily imparting the malt aroma and caramel flavour. (E,E)-2,4-Sebacedienal is the pyrolysis product of 1,2-linolenic acid. Heptanal and hexanal-saturated aliphatic aldehydes are the hydroperoxide decomposition products of 1,1-linoleic acid and 1,3-linoleic acid, respectively, thus imparting the green aroma and fruit flavour. Aromatic aldehydes such as benzene acetaldehyde and heterocyclic furfural are derived from Streker degradation of amino acids in the Maillard reaction, while saturated aliphatic aldehydes such as heptyl aldehyde and hexanal can provide the food bread flavour and fruit flavour, respectively.

However, trans C18:3 is slightly negatively correlated with 2,5-dimethyl-4-hydroxy-3(2H)-furanone, which imparts the caramel sweet flavour, concurrent with the preferences of the consumers. This substance is mostly derived from the Maillard reaction and has only a slight association with the generation of TFAs.

\section{Conclusions}

This study is the first to carry out PLSR analysis to analyse the association between TFAs and key flavour compounds in youtiao. Our results show that C18:2, 9t12t levels were significantly positively correlated with levels of trans-2- 
hexenal and nonaldehyde. These compounds play an important role in the formation of the flavour profile of youtiao and are an essential component of the characteristic flavour of youtiao.

C18:2, 9c12t and C18:2, 9t12c levels were significantly positively correlated with those of (E,E)-2,4-sebacedienal. The high FD value and content of (E,E)-2,4-sebacedienal are essential for the characteristic flavour profile of youtiao.

Trans C18:3 levels were significantly positively correlated with most aliphatic aldehydes and pyrazines, which are essential components in the characteristic flavour of youtiao, primarily imparting malt aroma and caramel aroma.

Together, our results show that the formation of key flavour substances in youtiao inevitably requires the synthesis of the aforementioned trans fatty acids, and further studies are required to investigate methods to inhibit the synthesis of TFAs without affecting the formation of key flavour substances in youtiao.

\section{Data Availability}

The data used to support the findings of this study have not been made available because the data were collected two years ago.

\section{Conflicts of Interest}

The authors declare that there are no conflicts of interest.

\section{Authors' Contributions}

Xuelian Yang contributed significantly to analysis and manuscript preparation; Xiangyu Zhang performed the experiment; Xuelian Yang, Jianchun Xie, and Dongdong Yang helped perform the analysis with constructive discussions; and Chengtao Wang contributed to the conception of the study.

\section{Acknowledgments}

This study was supported by the National Key Research and Development Program of China (2017YFD0400106) and the Science and Technology Program of Beijing (Z171100001317004).

\section{References}

[1] H. Hua, X. Jiang, and S. Wu, "Validation and comparable analysis of aluminum in the popular Chinese fried bread youtiao by wavelength dispersive XRF," Food Chemistry, vol. 207, pp. 1-5, 2016.

[2] G. Li, S. Wu, L. Wang, and C. C. Akoh, "Concentration, dietary exposure and health risk estimation of polycyclic aromatic hydrocarbons (PAHs) in youtiao, a Chinese traditional fried food," Food Control, vol. 59, pp. 328-336, 2016.

[3] I. Jerković, J. Mastelić, and S. Tartaglia, "A study of volatile flavour substances in Dalmatian traditional smoked ham: impact of dry-curing and frying," Food Chemistry, vol. 104, pp. 1030-1039, 2007.
[4] Q. Zhang, C. Wan, C. Wang et al., "Evaluation of the nonaldehyde volatile compounds formed during deep-fat frying process," Food Chemistry, vol. 243, pp. 151-161, 2018.

[5] S. Kesen, "Characterization of aroma and aroma-active compounds of Turkish turmeric (Curcuma longa) extract," Journal of Raw Materials to Processed Foods, vol. 1, pp. 13-21, 2020.

[6] N. R. Breternitz, H. M. A. Bolini, and M. D. Hubinger, "Sensory acceptance evaluation of a new food flavoring produced by microencapsulation of a mussel (Perna perna) protein hydrolysate," LWT - Food Science and Technology, vol. 83, pp. 141-149, 2017.

[7] H. Tian, P. Wang, P. Zhan, H. Yan, W. Zhou, and F. Zhang, "Effects of $\beta$-glucosidase on the aroma characteristics of flat peach juice as assessed by descriptive sensory analysis and gas chromatography and compared by partial least squares regression," LWT-Food Science and Technology, vol. 82, pp. 113-120, 2017.

[8] P. Wang, P. Zhan, H. Tian, F. Zhang, and J. Xi, "Characterization of the influence of thermal sterilization on the volatiles in flat peach juice," Analytical Letters, vol. 51, no. 15, pp. 2340-2350, 2018.

[9] R. G. Buttery, W. J. Orts, G. R. Takeoka, and Y. Nam, "Volatile flavor components of rice cakes," Journal of Agricultural and Food Chemistry, vol. 47, no. 10, pp. 4353-4356, 1999.

[10] S. N. Lotfy, H. H. M. Fadel, A. H. El-Ghorab, and M. S. Shaheen, "Stability of encapsulated beef-like flavourings prepared from enzymatically hydrolysed mushroom proteins with other precursors under conventional and microwave heating," Food Chemistry, vol. 187, pp. 7-13, 2015.

[11] B. Zhou, Y. Zhao, X. Wang, D. Fan, K. Cheng, and M. Wang, "Unraveling the inhibitory effect of dihydromyricetin on heterocyclic aromatic amines formation," Journal of the Science of Food and Agriculture, vol. 98, no. 5, pp. 1988-1994, 2018.

[12] Q. Zhou, C. L. Wintersteen, and K. R. Cadwallader, "Identification and quantification of aroma-active components that contribute to the distinct malty flavor of buckwheat honey," Journal of Agricultural and Food Chemistry, vol. 50, no. 7, pp. 2016-2021, 2002.

[13] O. Nashalian, X. Wang, and V. A. Yaylayan, "Formation of the reduced form of furaneol (2,5-dimethyl-4-hydroxy-tetrahydrofuran-3-one) during the Maillard reaction through catalysis of amino acid metal salts," Food Chemistry, vol. 210, pp. 43-48, 2016.

[14] J. Pico, J. Bernal, and M. Gómez, "Wheat bread aroma compounds in crumb and crust: a review," Food Research International, vol. 75, pp. 200-215, 2015.

[15] Y. Wang, H. Rodolfo Juliani, J. E. Simon, and C.-T. Ho, "Amino acid-dependent formation pathways of 2-acetylfuran and 2,5-dimethyl-4-hydroxy-3[2H]-furanone in the Maillard reaction," Food Chemistry, vol. 115, no. 1, pp. 233-237, 2009.

[16] H. Yahya, R. S. T. Linforth, and D. J. Cook, "Flavour generation during commercial barley and malt roasting operations: a time course study," Food Chemistry, vol. 145, pp. 378-387, 2014.

[17] M. Zhang, X. Chen, K. Hayat et al., "Characterization of odoractive compounds of chicken broth and improved flavor by thermal modulation in electrical stewpots," Food Research International, vol. 109, pp. 72-81, 2018.

[18] M. A. Majcher, D. Klensporf-Pawlik, M. Dziadas, and H. H. Jeleń, "Identification of aroma active compounds of cereal coffee brew and its roasted ingredients," Journal of 
Agricultural and Food Chemistry, vol. 61, no. 11, pp. 26482654, 2013.

[19] P. Rotsatchakul, S. Chaiseri, and K. R. Cadwallader, "Identification of characteristic aroma components of Thai fried chili paste," Journal of Agricultural and Food Chemistry, vol. 56, no. 2, pp. 528-536, 2008.

[20] Y. Takakura, T. Sakamoto, S. Hirai, T. Masuzawa, H. Wakabayashi, and T. Nishimura, "Characterization of the key aroma compounds in beef extract using aroma extract dilution analysis," Meat Science, vol. 97, no. 1, pp. 27-31, 2014.

[21] Y. Zhang, M. Huang, H. Tian, B. Sun, J. Wang, and Q. Li, "Preparation and aroma analysis of Chinese traditional fermented flour paste," Food Science and Biotechnology, vol. 23, no. 1, pp. 49-58, 2014.

[22] Y. Li, Y. Yang, and A.-N. Yu, "Effects of reaction parameters on generation of volatile compounds from the Maillard reaction between L-ascorbic acid and glycine," International Journal of Food Science \& Technology, vol. 51, no. 6, pp. 1349-1359, 2016.

[23] Y. Zhang, R. Lu, H. Song, S. Zheng, Y. Chen, and N. Zhou, "Effect of stir frying on the antioxidant capacity and aroma components of bok choy (Chinese cabbage)," International Journal of Food Properties, vol. 19, no. 11, pp. 2536-2549, 2016.

[24] X. X. Mo, "Rapid quantitative detection and model optimization of trans fatty acids in edible vegetable oils by near infrared spectroscopy," Chinese Journal of Analytical Chemistry, vol. 11, pp. 1694-1702, 2017.

[25] E. S. Erten and K. R. Cadwallader, "Identification of predominant aroma components of raw, dry roasted and oil roasted almonds," Food Chemistry, vol. 217, pp. 244-253, 2017.

[26] C. Dobarganes, G. Márquez-Ruiz, and J. Velasco, "Interactions between fat and food during deep-frying," European Journal of Lipid Science and Technology, vol. 102, no. 8-9, pp. 521-528, 2000.

[27] R. G. Buttery and L. C. Ling, "Volatile flavor components of corn tortillas and related products," Journal of Agricultural and Food Chemistry, vol. 43, no. 7, pp. 1878-1882, 1995.

[28] S. Song, "Rapid measuring and modelling flavour quality changes of oxidised chicken fat by electronic nose profiles through the partial least squares regression analysis," Food Chemistry, vol. 141, no. 4, pp. 4278-4288, 2013. 